G. Ikegami

Nagoya Math. J.

Vol. 90 (1983), 63-76

\title{
ON EXISTENCE OF TOLERANCE STABLE DIFFEOMORPHISMS*
}

\author{
GIKŌ IKEGAMI
}

\section{§ 1. Introduction}

We consider a compact smooth manifold $M$. Diff ${ }^{1}(M)$ denotes the space of $C^{1}$-diffeomorphisms of $M$ onto itself with the usual $C^{1}$-topology. In the research of the qualitative theory of dynamical systems there is a desire to find a concept of stability of geometric global structure of orbits such that this stable systems are dense in the space of dynamical systems on $M$. Structural stability does not satisfy the density condition in $\operatorname{Diff}^{1}(M)$. Tolerance stability (see Section 2 for definition) is a candidate for the density property [7, p. 294]. Concerning tolerance stability there are researches as [6], [7], [8], and [2].

If $f \in \operatorname{Diff}^{1}(M)$ is structurally stable in strong sense, $f$ is topologically stable in $\operatorname{Diff}^{1}(M)$ (see Section 2 for definition). Moreover, topological stability implies tolerance stability [A. Morimoto, 2]. The proof of this property will be introduced in Section 2 .

The main result of this paper is to show the existence of diffeomorphisms on any compact manifold $M$ which are tolerance stable but not topologically stable in $\operatorname{Diff}^{1}(M)$, so that, not structurally stable in strong sense. This will be proved in Sections 3,4 and 5 .

\section{$\S 2$. Definitions and statement of results}

We denote by Homeo $(M)$ the set of homeomorphisms of $M$ onto itself; the topology on Homeo $(M)$ is given by the neighborhood $N_{\mathrm{s}}(f)$ of $f \in$ Homeo $(M)$

$$
N_{\varepsilon}(f)=\{g ; d(f, g)<\varepsilon\}, \quad \varepsilon>0 .
$$

Here, for a Riemannian metric $d$ on $M, d(f, g)<\varepsilon$ means

Received October 12, 1981.

* The author is partly supported by Grant in Aid for Scientific Research Project No. 546004 . 


$$
d(f(x), g(x))<\varepsilon \quad \text { for } x \in M .
$$

To state the definition of tolerance stability, we need the following definition:

Definition (2.1). $f, g \in \operatorname{Homeo}(M)$ are orbit- $\varepsilon$-equivalent, $\varepsilon>0$, if 1. for every $f$-orbit $O_{f}$, there is a g-orbit $O_{g}$ such that

(a) $O_{f} \subset U_{\varepsilon}\left(O_{g}\right)$

(b) $O_{g} \subset U_{\varepsilon}\left(O_{f}\right)$, and

2. for every g-orbit $O_{g}$, there is a f-orbit $O_{f}$ such that

(a) $O_{g} \subset U_{s}\left(O_{f}\right)$

(b) $O_{f} \subset U_{\varepsilon}\left(O_{g}\right)$.

Here, $U_{\varepsilon}(*)$ is the $\varepsilon$-neighborhood of $*$.

Suppose that a subset $\mathscr{D}$ of Homeo $(M)$ is given a topology not coarser than that of Homeo $(M)$.

Definition (2.2). An element $f \in \mathscr{D}$ is tolerance-stable in $\mathscr{D}$ if for every $\varepsilon>0$ there is a neighborhood $N$ of $f$ in $\mathscr{D}$ (with respect to the given topology on $\mathscr{D})$ such that, for every $g \in N, f$ and $g$ are orbit- $\varepsilon$-equivalent.

Definition (2.3). An element $f \in \mathscr{D}$ is topologically stable in $\mathscr{D}$, if for any $\varepsilon>0$ there is a neighborhood $N$ of $f$ in $\mathscr{D}$ such that for every $g \in N$ there is a continuous map $h: M \rightarrow M$ satisfying

(a) $d\left(h, i_{M}\right)<\varepsilon$, where $i_{M}$ is the identity map of $M$,

(b) $h g=f h$.

The following property is mentioned and proved by A. Morimoto in [2]. We introduce this:

Proposition. If $M$ is a compact topological manifold and $f \in \operatorname{Homeo}(M)$ is topologically stable in $\mathscr{D}$ then $f$ is tolerance stable in $\mathscr{D}$, for any subset $\mathscr{D} \subset$ Homeo $(M)$.

Proof. For closed non-empty subsets $A$ and $B$ of $M$, let

$$
\bar{d}(A, B)=\max \left\{\max _{a \in A} d(a, B), \max _{b \in B} d(b, A)\right\},
$$

where $d(a, B)=\min _{b \in B} d(a, b) . \quad O_{f}(x)$ denotes the f-orbit of $x ; O_{f}(x)=$ $\left\{f^{i}(x) ; i \in Z\right\}$. Put $\bar{O}_{f}(x)=\mathrm{Cl}\left(O_{f}(x)\right)$. By the assumption, for every $\varepsilon>0$, 
there is a neighborhood $N$ of $f$ in $\mathscr{D}$ such that for every $g \in N$ there is $h: M \rightarrow M$ satisfying (a) and (b) in Definition (2.3). By (b), $h\left(O_{g}(x)\right)=$ $O_{f}(h(x))$ for every $x \in M$. Hence,

$$
\bar{d}\left(\bar{O}_{g}(x), \bar{O}_{f}(h(x))\right)=\bar{d}\left(\bar{O}_{g}(x), h\left(\bar{O}_{g}(x)\right)\right)<\varepsilon .
$$

Therefore, for any g-orbit $O_{g}$ there is f-orbit $O_{f}$ such that $O_{g} \subset U_{s}\left(O_{f}\right)$. and $O_{f} \subset U_{s}\left(O_{g}\right)$. Since $M$ is a compact manifold, we can prove that $d\left(h, i_{M}\right)<\varepsilon$ implies that $h: M \rightarrow M$ is a surjection if $\varepsilon>0$ is sufficiently small. We may assume that $\varepsilon$ is taken so small that this property is satisfied. Hence for every $x \in M$ there is $y \in M$ such that $h(y)=x$. Then

$$
\begin{aligned}
\bar{d}\left(\bar{O}_{f}(x), \bar{O}_{g}(y)\right) & =\bar{d}\left(\bar{O}_{f}(h(y)), \bar{O}_{g}(y)\right) \\
& =\bar{d}\left(h\left(\bar{O}_{g}(y)\right), \bar{O}_{g}(y)\right)<\varepsilon .
\end{aligned}
$$

Hence, for any $f$-orbit $O_{f}$ there is g-orbit $O_{g}$ such that $O_{f} \subset U_{\varepsilon}\left(O_{g}\right)$ and $O_{g} \subset U_{\varepsilon}\left(O_{f}\right)$. Therefore, $f$ is tolerance stable in $\mathscr{D}$.

Definition (2.4). Two elements $f, g \in \operatorname{Diff}^{1}(M)$ are topologically $\varepsilon$ conjugate if there is a homeomorphism $h: M \rightarrow M$ such that $h g=f h$ and $d(h(x), x)<\varepsilon$ for every $x \in M$. $f, g$ are topologically conjugate if there is a homeomorphism $h$ such that $h g=f h$.

Definition (2.5). An element $f \in \operatorname{Diff}^{1}(M)$ is structurally stable in strong sense if for every $\varepsilon>0$ there is a neighborhood $N$ of $f$ in $\operatorname{Diff}^{1}(M)$ such that every $g \in N$ are topologically $\varepsilon$-conjugate to $f . f$ is structurally stable, if there is $N$ such that, for every $g \in N, f$ and $g$ are topologically conjugate.

Structural stability in strong sense implies structural stability and topological stability in $\operatorname{Diff}^{1}(M)$. If $f \in \operatorname{Diff}^{1}(M)$ satisfies Axiom $A$ and strong transversality condition then $f$ is structurally stable in strong sense [4].

THEOREM. Let $M$ be a compact differentiable manifold. There is a diffeomorphism $f$, in the boundary $\partial \Sigma$ of the set $\Sigma$ of all structurally stable elements in $\operatorname{Diff}^{1}(M)$, such that

(a) $f$ is tolerance-stable in $\operatorname{Diff}^{1}(M)$, and

(b) $f$ is not topologically stable in $\operatorname{Diff}^{1}(M)$, so that, $f$ is not structurally stable in strong sense. 


\section{§3. Construction of $f$}

Theorem is proved in Sections 3, 4 and 5. In these sections $M$ is assumed to have $\operatorname{dim} M \geqq 2$. But to the readers of these sections the proof of Theorem in the case $\operatorname{dim} M=1$ will be obvious.

$f$ will be constructed as follows. If $f_{0}$ is a diffeomorphism which is structurally stable in strong sense and has a periodic point $p$ that is a sink or source, then $f$ will be obtained from $f_{0}$ by isotopically replacing $f_{0}$ on a small neighborhood of $p$.

Let $f_{0}$ be a time-one map of the flow of the vector field $Y$ obtained by Theorem 2.1 of [5]. Then $f_{0}$ is a Morse-Smale diffeomorphism having a fixed point $p$ which is a sink. By [3], $f_{0}$ is structurally stable in strong sense.

By replacing $f_{0}$ by an isotopy on a small neighborhood $U$ of $p$ we obtain $f_{1}$ such that

(i) every point in a small closed ball neighborhood $B$ in $U$, with center $p$, is a fixed point of $f_{1}$, and

(ii) for every $x$ in $U-B, \lim _{k \rightarrow \infty} f_{1}^{k}(x)$ exists in $\partial B$.

Let $B_{r}$ be a closed ball in the euclidean space $\boldsymbol{R}^{m}$ of the same dimension as $M$, centered on the origin with radius $r$. Let $S_{r}=\partial B_{r}, a(m-1)$ sphere. After this, we regard $B$ as a closed ball $B_{r_{0}}$ in $\boldsymbol{R}^{m}$, and $p$ as the origin of $\boldsymbol{R}^{m}$.

To construct $f$ we will define a vector field $V$ on $B$. On a neighborhood of $p, f$ will be the time-one map of the flow of $V$.

(1) Construction of $V$.

For this purpose we at first define a vector field $X$. Let

$$
\varphi_{0}(r)=\mathrm{e}^{-1 / r^{2}} \sin \frac{1}{r}, \quad r>0 .
$$

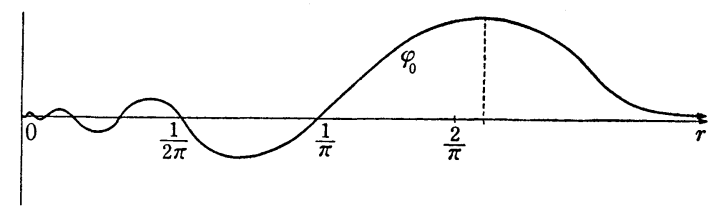

Fig. 1.

Take $r_{1} \in \boldsymbol{R}_{+}$such that $r_{1}<r_{0}, \varphi_{0}^{\prime}\left(r_{1}\right)>0$, and

$$
\frac{1}{2 n \pi}<r_{1}<\frac{1}{(2 n-1) \pi} \quad \text { for a fixed } n \in Z_{+} .
$$


Let $\alpha:\left[r_{1}, \infty\right) \rightarrow R$ be a $C^{1}$-function such that $\alpha(r)<0$ and $\alpha^{\prime}(r)>0$ for every $r \in\left[r_{1}, \infty\right)$, and that the function defined by

$$
\varphi(r)= \begin{cases}0 & \text { if } r=0 \\ \varphi_{0}(r) & \text { if } 0<r<r_{1} \\ \alpha(r) & \text { if } r_{1} \leqq r\end{cases}
$$

is $C^{1}$.

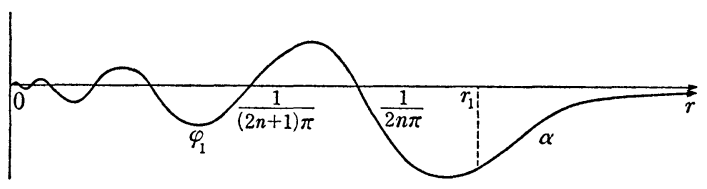

Fig. 2.

Define a vector field $X$ on $B$ by

$$
X_{x}= \begin{cases}\varphi(\|x\|) \frac{x}{\|x\|} & \text { if } x \neq 0 \\ 0 & \text { if } x=0\end{cases}
$$

Here, $\|\cdot\|$ is the euclidean norm on $\boldsymbol{R}^{m}$.

We show that $X$ is $C^{1}$. Let $x={ }^{t}\left(x_{1}, \cdots, x_{m}\right) \in \boldsymbol{R}^{m}$ be a row vector, i.e. the transposition of $\left(x_{1}, \cdots, x_{m}\right)$. If $x \neq 0$

$$
\begin{aligned}
\frac{\partial}{\partial x_{i}} X_{x} & =\frac{\partial}{\partial x_{i}}\left(\frac{\varphi(\|x\|)}{\|x\|}\right) x+\frac{\varphi(\|x\|)}{\|x\|} \frac{\partial}{\partial x_{i}} x \\
& =\frac{\partial}{\partial x_{i}}\|x\| \frac{\varphi^{\prime}(\|x\|)\|x\|-\varphi(\|x\|)}{\|x\|^{2}} x+\frac{\varphi(\|x\|)}{\|x\|} \frac{\partial}{\partial x_{i}} x \\
& =\frac{x_{i}}{\|x\|} \frac{\varphi^{\prime}(\|x\|)\|x\|-\varphi(\|x\|)}{\|x\|^{2}} x+\frac{\varphi(\|x\|)}{\|x\|} \frac{\partial}{\partial x_{i}} x \\
& =x_{i}\left(\frac{\varphi^{\prime}(\|x\|)}{\|x\|^{2}}-\frac{\varphi(\|x\|)}{\|x\|^{3}}\right) x+\frac{\varphi(\|x\|)}{\|x\|} \frac{\partial}{\partial x_{i}} x .
\end{aligned}
$$

Hence, for $x \neq 0$

$$
D X_{x}=\left(\frac{\varphi^{\prime}(\|x\|)}{\|x\|^{2}}-\frac{\varphi(\|x\|)}{\|x\|^{3}}\right) x \cdot{ }^{t} x+\frac{\varphi(\|x\|)}{\|x\|} I
$$

where $D X_{x}$ is the Jacobian matrix, and $I$ is the unit matrix. For a matrix $A=\left(a_{1}, \cdots, a_{m}\right)$ with row vectors $a_{1}, \cdots, a_{m}$, we define the norm of $A$ by 


$$
\|A\|=\max _{j}\left\|a_{j}\right\|
$$

Then,

$$
\left\|D X_{x}\right\| \leqq\left|\frac{\varphi^{\prime}(\|x\|)}{\|x\|^{2}}-\frac{\varphi(\|x\|)}{\|x\|^{3}}\right| \cdot\|x\|^{2}+\left|\frac{\varphi(\|x\|)}{\|x\|}\right|
$$

$D X_{0}=0$ since $\varphi^{\prime}(0)=0$. Therefore, since $\varphi$ is $C^{1}, X$ is a $C^{1}$-vector field.

Next we define a vector field $Y$ on $B$. Let $\mu:[0, \infty) \rightarrow[0, \infty)$ be a $C^{1}$-function such that

$$
\left\{\begin{array}{l}
\mu \geqq 0, \quad \text { and } \\
\mu(r)=0 \quad \text { and } \quad \mu^{\prime}(r)=0 \quad \text { if } r=0 \text { or } r \geqq r_{1} .
\end{array}\right.
$$

Let $C$ be a $C^{1}$-vector field, on the unit sphere $S^{m-1}$, such that $C$ has two singular points $p_{+}$and $p_{-}$, where $p_{+}$is a source at the north pole and $p_{-}$is a sink at the south pole, and such that every other trajectory of $C$ goes out of $p_{+}$and into $p_{-}$. Then $Y$ is defined by

$$
Y_{x}= \begin{cases}\mu(\|x\|) C_{x /\|x\|} & \text { if } x \neq 0 \\ 0 & \text { if } x=0 .\end{cases}
$$

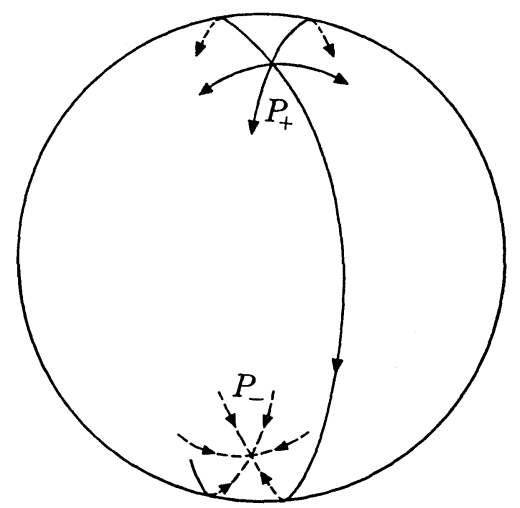

Fig. 3.

For the calculation of the derivative of $Y_{x}$, we take a $C^{1}$-extension $\tilde{C}: U\left(S^{m-1}\right) \rightarrow R^{m}$ of $C: S^{m-1} \rightarrow R^{m}$, where $U\left(S^{m-1}\right)$ is a neighborhood of $S^{m-1}$ in $\boldsymbol{R}^{m}$. Then, for $x \neq 0$, we have

$$
\mu(\|x\|) C_{x /\|x\|}=\mu(\|x\|) \tilde{C}_{x /\|x\|} .
$$

Let $e_{i}$ be the $i$-th row vector of the unit matrix $I$. Let $y=x /\|x\|$, and let $D$ be the notation of the derivative of variable $x$. Since 


$$
\begin{aligned}
& \frac{\partial}{\partial x_{i}} \frac{x}{\|x\|}=-\frac{x_{i}}{\|x\|^{3}} x+\frac{1}{\|x\|} e_{i}, \quad \text { and } \\
& D Y_{x}=D \mu(\|x\|) \cdot \tilde{C}_{y}+\mu(\|x\|) \cdot D \tilde{C}_{y} \cdot D\left(\frac{x}{\|x\|}\right),
\end{aligned}
$$

we have

$$
\begin{aligned}
\frac{\partial}{\partial x_{i}} Y_{x} & =\frac{\partial}{\partial x_{i}}(\mu(\|x\|)) \tilde{C}_{y}+\mu(\|x\|) \cdot D \tilde{C}_{y} \cdot \frac{\partial}{\partial x_{i}} \frac{x}{\|x\|} \\
& =\frac{x_{i}}{\|x\|} \mu^{\prime}(\|x\|) \tilde{C}_{y}+\mu(\|x\|) \cdot D \tilde{C}_{y} \cdot\left(-\frac{x_{i}}{\|x\|^{3}} x+\frac{1}{\|x\|} e_{i}\right) .
\end{aligned}
$$

Consequently, if $x \neq 0$ then

$$
D Y_{x}=\mu^{\prime}(\|x\|) \tilde{C}_{y} \cdot t_{y}+\mu(\|x\|) \cdot D \tilde{C}_{y} \cdot\left(-\frac{1}{\|x\|^{3}} x \cdot{ }^{t} x+\frac{1}{\|x\|} I\right) .
$$

Since $\mu(0)=\mu^{\prime}(0)=0$ we have $D Y_{0}=0$. Therefore $Y$ is a $C^{1}$-vector field. The $C^{1}$-vector field $V$ on $B$ is defined by

$$
V_{x}=X_{x}+Y_{x}
$$

Fig. 4 shows the orbit structure of $V$. Here, we denote $B(k)=B_{1 / k \pi}$ and $S(k)=\partial B(k)$. Every singular point of $V$ is hyperbolic except $p$.

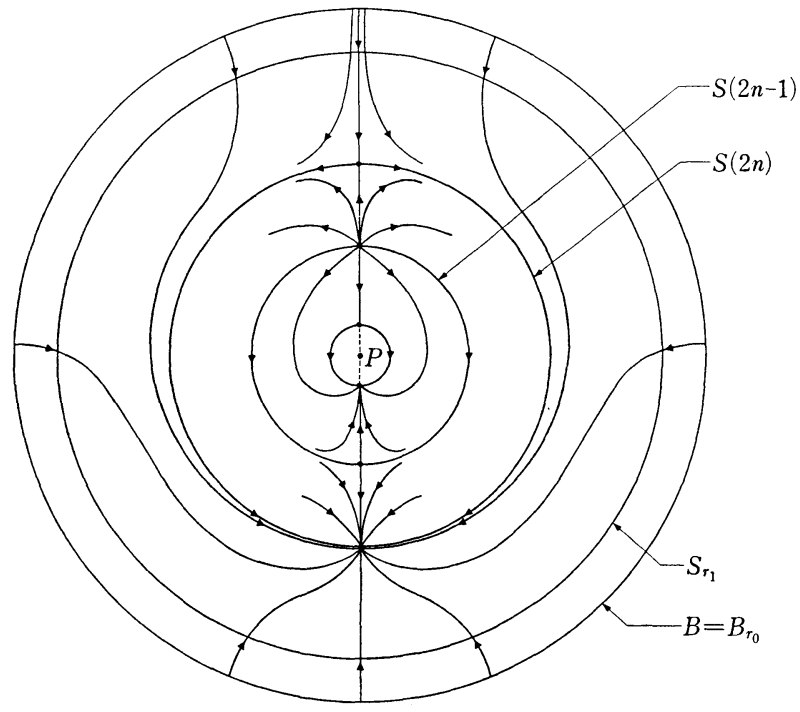

Fig. 4. 
(2) Construction of $f$.

Let $\Psi_{1}: B \rightarrow B$ be the time one map of the flow $\Psi$ of $V . \Psi_{1}$ is a $C^{1}$ diffeomorphism such that $B-\Psi_{1}(B)$ is an annulus which is diffeomorphic to $\partial B \times[0,1)$. Every fixed point of $\Psi_{1}$ is hyperbolic except $p$. The property (ii) of $f_{1}$ and the orbit structure of $V$ enable us to obtain a diffeomorphism $f: M \rightarrow M$ satisfying the following properties;

(i) $f \mid B=\Psi_{1}$,

(ii) $f \mid(M-U)=f_{1}(M-U)$,

(iii) if $x \in U-B$ then $\lim _{k \rightarrow \infty} f^{k}(x)$ is the north pole or the south pole of $S(2 n)$.

Moreover, $f \mid(M-B)$ is obtained from $f_{1}$ by an isotopy supported by $U$. Since $\Psi_{1}$ is isotopic to $i_{B}=f_{1} \mid B$ by the isotopy $\Psi_{t}, t \in[0,1], f$ is isotopic to $f_{1}$ by an isotopy supported by $U$.

In Sections 4 and 5 it is proved that $f$ possesses the desired properties (a), (b) of Theorem.

§4. Proof of tolerance-stability of $f$ in $\operatorname{Diff}^{1}(M)$

Let sufficiently small $\varepsilon>0$ be given.

Lemma. There is a diffeomorphism $h: M \rightarrow M$ such that (i) $h=$ identity on $M-B_{\varepsilon / 4}$, and (ii) $f_{\varepsilon}=h f$ is structurally stable in strong sense.

Proof. We may assume

$$
\frac{\varepsilon}{3}<r_{1}
$$

Let $\ell$ be a sufficiently large integer satisfying the following inequalities.

$$
\frac{1}{2 \ell \pi}+\mathrm{e}^{-(\ell \pi)^{2}}<\frac{1}{(2 \ell-1) \pi}<\frac{\varepsilon}{4} .
$$

Put $1 / 2 \ell \pi+\mathrm{e}^{-(\ell \pi)^{2}}=r_{2}$. Define a discontinuous function $\eta_{0}:\left(0, r_{2}\right) \rightarrow \boldsymbol{R}_{+}$by

$$
\eta_{0}(r)= \begin{cases}r-\mathrm{e}^{-(k \pi)^{2}} & \text { if } \frac{1}{(k+1) \pi}<r \leqq \frac{1}{k \pi}, \\ r-\mathrm{e}^{-4(2 \ell \pi)^{2}} & \text { if } \frac{1}{2 \ell \pi}<r \leqq r_{2},\end{cases}
$$

where $k=2 \ell, 2 \ell+1,2 \ell+3, \cdots$ Let $\eta: \boldsymbol{R}_{+} \rightarrow \boldsymbol{R}_{+}$be a $C^{1}$-function satisfying 


$$
\begin{cases}0 \leqq \eta(r) \leqq r, & \\ \eta(r)=r & \text { if } r>\frac{1}{(2 \ell-1) \pi}, \\ \eta(r)<\eta_{0}(r) & \text { if } 0<r \leqq r_{2}, \\ \eta(0)=0, & \text { for every } r \geqq 0, \\ \eta^{\prime}(r)>0 & \\ \eta^{\prime}(0)<1 . & \end{cases}
$$

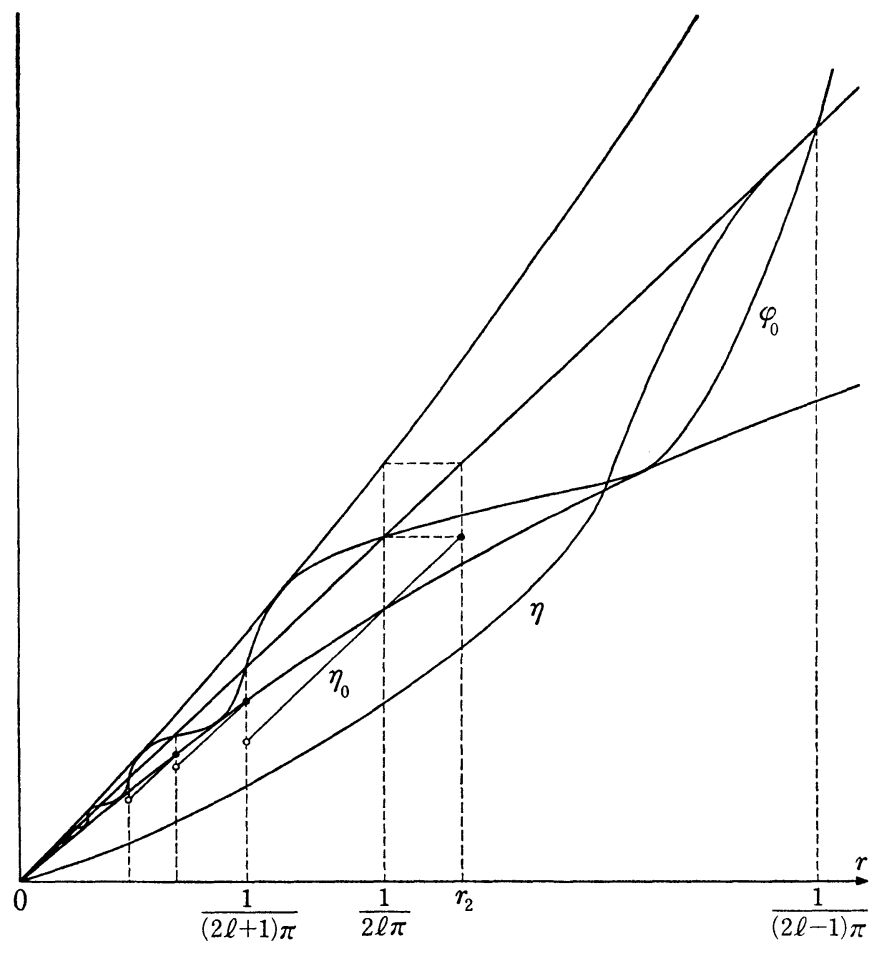

Fig. 5.

In fact, $\eta$ exists. Especially we can find $\eta$ such that $0<\eta^{\prime}(0)<1$, since in a neighborhood of 0 the following properties hold.

$$
\eta_{0}(r)>r-\mathrm{e}^{-(1 / r-\pi)^{2}},
$$

$$
\lim _{r \rightarrow 0} \frac{1}{r}\left(r-\mathrm{e}^{-(1 / r-\pi)^{2}}\right)=1 \text {. }
$$

Define $h: M \rightarrow M$ by 


$$
h(x)= \begin{cases}\eta(\|x\|) \frac{x}{\|x\|} & \text { if } x \in B \\ x & \text { if } x \notin B .\end{cases}
$$

Since $B=B_{r_{0}}$ and $r_{1}<r_{0}$, the map $h$ is well defined by (4.1), (4.2) and (4.3). $h$ is a diffeomorphism. Define $f_{s}$ by

$$
f_{\varepsilon}(x)=h f(x) .
$$

By $(4.3) f_{s}(x)=f(x)$ if $\|x\| \geqq 1 /(2 \ell-1) \pi$.

Next, we show

$$
\left\|f_{s}(x)\right\|<\|x\| \quad \text { if }\|x\|<\frac{1}{(2 \ell-1) \pi} .
$$

Remember the definition of the vector field $X$, then we observe that $\|f(x)\| \leqq\|x\|$ when $1 / 2 k \pi<\|x\|<1 /(2 \ell-1) \pi$. Since $\eta(\|x\|) \leqq\|x\|$, it follows that

$$
\left\|f_{s}(x)\right\|<\|x\| \quad \text { if } \frac{1}{2 k \pi}<\|x\|<\frac{1}{(2 k-1) \pi}, \quad k \geqq \ell .
$$

Next, let $1 /(2 k+1) \pi<\|x\|<1 / 2 k \pi$. Let $\bar{\Psi}_{t}(x)$ be the flow of $X$, so that $\bar{\Psi}_{0}(x)=x$. Since $V_{x}=X_{x}+Y_{x}$ and $\|f(x)\|=\left\|\Psi_{1}(x)\right\|=\left\|\bar{\Psi}_{1}(x)\right\|$, we have

$$
\|f(x)\|=\|x\|+\int_{0}^{1} \varphi\left(\left\|\bar{\Psi}_{t}(x)\right\|\right) d t,
$$

where $\varphi(r)=\mathrm{e}^{-1 / r^{2}} \sin 1 / r$ as before. $1 /(2 k+1) \pi \leqq\|x\| \leqq 1 / 2 k \pi$ implies $0 \leqq \sin (1 /\|x\|) \leqq 1$.

Hence,

$$
\varphi(\|x\|) \leqq \mathrm{e}^{-1 /\|x\|^{2}} \leqq \mathrm{e}^{-(2 k \pi)^{2}} .
$$

Therefore, by (4.9),

$$
\|f(x)\| \leqq\|x\|+\mathrm{e}^{-(2 k \pi)^{2}} .
$$

Using this and the definition of $\eta_{0}$ we have

$$
\begin{aligned}
\left\|f_{c}(x)\right\| & =\|h f(x)\|=\eta(\|f(x)\|) \\
& \leqq \eta\left(\|x\|+\mathrm{e}^{\left.-(2 k \pi)^{2}\right)}<\eta_{0}\left(\|x\|+\mathrm{e}^{-(2 k \pi)^{2}}\right)\right. \\
& <\left(\|x\|+\mathrm{e}^{-(2 k \pi)^{2}}\right)-\mathrm{e}^{-(2 k \pi)^{2}}=\|x\| .
\end{aligned}
$$

Hence, 


$$
\left\|f_{\varepsilon}(x)\right\|<\|x\| \quad \text { if } \frac{1}{(2 k+1) \pi} \leqq\|x\| \leqq \frac{1}{2 k \pi}
$$

By (4.8) and (4.10) we have (4.7).

Hence $f_{\varepsilon}$ contracts to $p$ in Int $B(2 \ell-1)$. We have $f_{\varepsilon}=f$ in $M-$ $B_{1 /(2 \ell-1) \pi}$ by (4.3). By the definition of $f, f \mid\left(M-B_{1 /(2 \ell-1) \pi}\right)$ is Morse-Smale and $\partial B_{1 /(2 \ell-1) \pi}$ is $f$-invariant. Therefore $f_{\varepsilon}$ is Morse-Smale. Since a MorseSmale diffeomorphism is structurally stable in strong sense by [3] this completes the proof of Lemma.

Since $f_{s}$ is structurally stable in strong sense there is a neighborhood $N_{0}$ of $f_{\varepsilon}$ in $\operatorname{Diff}^{1}(M)$ such that every element in $N_{0}$ is topologically $\varepsilon / 24$ conjugate to $f_{s}$.

Since $h$ is a $C^{1}$-diffeomorphism the map $h_{*}: \operatorname{Diff}^{1}(M) \rightarrow \operatorname{Diff}^{1}(M)$ defined by $h_{*}(g)=h g$ is continuous [1, p. 229, (B.8)]. Hence, for the neighborhood $N_{0}$ of $h f=f_{s}$, there is a neighborhood $N$ of $f$ in $\operatorname{Diff}^{1}(M)$ such that

$$
g \in N \Rightarrow h g=g_{\varepsilon} \in N_{0} \text {. }
$$

Hereafter, let $g$ be included in $N$. Since $h=$ identity on $M-B_{\varepsilon / 4}$ by (4.2), (4.3) and (4.6), we have

$$
\begin{aligned}
& f_{\varepsilon}=f \text { and } g_{\varepsilon}=g \text { in } M-B_{\varepsilon / 4} \\
& f_{\varepsilon} \text { and } g_{\varepsilon} \text { are topologically } \varepsilon / 24 \text {-conjugate: }
\end{aligned}
$$

there is a homeomorphism $h_{g}: M \rightarrow M$ such that

$$
h_{g} g_{\varepsilon}=f_{\varepsilon} h_{g} \text { and } d\left(h_{g}(x), x\right)<\varepsilon / 24, \quad \text { for all } x .
$$

We may assume that $\varepsilon$ is small, so that there is an integer $k$ satisfying $3 / \pi \varepsilon<k<24 / 7 \pi \varepsilon$. Then we have

$$
\frac{\varepsilon}{4}+\frac{\varepsilon}{24}<\frac{1}{k \pi}<\frac{\varepsilon}{3}
$$

(4.1), (4.13) and the definition of $f$ imply that $S_{1 / k \pi}$ is $f$-invariant. Denote $S_{f}=S_{1 / k \pi}$. Since $S_{f}$ is contained in the complement of $B_{\varepsilon / 4}$, (4.11) implies that $S_{f}$ is also $f_{\mathrm{s}}$-invariant. Since $f_{\varepsilon}$ and $g_{\varepsilon}$ are topologically $\varepsilon / 24$-conjugate, (4.11) and (4.13) imply that $h_{g}\left(S_{f}\right)$ is contained in $M-B_{\varepsilon / 4}$ and is both $g$ and $g_{s}$-invariant. Denote $h_{g}\left(S_{f}\right)=S_{g}, B_{1 / k \pi}=B_{f}$ and $h_{g}\left(B_{f}\right)=B_{g}$. Since $\partial B_{f}=S_{f}$ and $\partial B_{g}=S_{g}$ we have 


$$
\left\{\begin{array}{l}
f_{\varepsilon}=f \quad \text { in } M-B_{f}, \\
g_{\varepsilon}=g \quad \text { in } M-B_{g}, \\
f \mid\left(M-B_{f}\right) \text { and } g \mid\left(M-B_{g}\right) \text { are topologically } \varepsilon / 24 \text {-conjugate. }
\end{array}\right.
$$

Precisely, the last part of (4.14) means that there is the commutative diagram

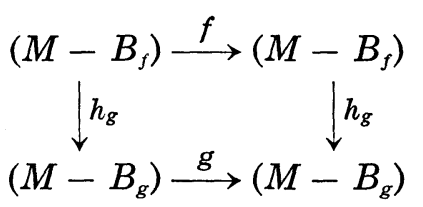

and $d\left(h_{g}(x), x\right)<\varepsilon / 24$ for every $x \in\left(M-B_{f}\right)$. (4.14) implies

$$
\begin{cases}B_{f} & \text { is } f \text {-invariant, } \\ B_{g} & \text { is g-invariant. }\end{cases}
$$

For every $g$ in $N$, we must show that $f$ and $g$ are orbit- $\varepsilon$-equivalent. First, let $O_{f} \subset M-B_{f}$. Then, $O_{f}$ is an $f_{\varepsilon}$-orbit $O_{f_{\varepsilon}}$. By (4.14), $h_{g}\left(O_{f}\right)=O_{g_{\varepsilon}}$ is contained in $M-B_{g}$ and $O_{g_{\varepsilon}}$ is a g-orbit $O_{g}$. Since $d\left(h_{g}, i_{M}\right)<\varepsilon / 24$ then the conditions (a) and (b) of 1 in Definition (2.1) are satisfied in this case.

Next, let $O_{f} \subset B_{f}$. Take any orbit $O_{g}$ in $B_{g}$ (by using (4.15)). Then (a) and (b) of 1 in Definition (2.1) are satisfied. In fact, for any $x \in B_{f}$ and $y \in B_{g}$, by (4.13) we have

$$
\begin{aligned}
\|x-y\| & \leqq\|x\|+\|y\| \\
& \leqq \frac{1}{k \pi}+\left(\frac{1}{k \pi}+\frac{\varepsilon}{24}\right) \\
& <\frac{\varepsilon}{3}+\left(\frac{\varepsilon}{3}+\frac{\varepsilon}{24}\right)<\varepsilon .
\end{aligned}
$$

Hence, the condition 1 in Definition (2.1) is satisfied. Similarly we can show the condition 2. Therefore $f$ is tolerance-stable in $\operatorname{Diff}^{1}(M)$.

\section{§5. Proof of topological unstability in $\operatorname{Diff}^{1}(M)$}

Suppose that $f$ is topologically stable in $\operatorname{Diff}^{1}(M)$. Then, for any $\varepsilon_{1}>0$ there is a neighborhood $N$ of $f$ in $\operatorname{Diff}^{1}(M)$ such that for every $g$ in $N$ there is a continuous map $\tau: M \rightarrow M$ satisfying

(a) $d\left(\tau, i_{M}\right)<\frac{\varepsilon_{1}}{2}$,

(b) $\tau g=f \tau$. 
For the fixed integer $n$ in (3.1), let

$$
\varepsilon_{1}=\frac{1}{2 n \pi} \text {. }
$$

To introduce a contradiction, we take following $g$;

$$
g=h f,
$$

where $h$ is a diffeomorphism defined by (4.6). But we must take $g$ such that $g \in N$. By (4.4), (4.5) and the definition (4.3) of $\eta$ we can choose $\eta$, by taking $\ell$ sufficiently large, such that $|\eta(r)-r|$ and $\left|\eta^{\prime}(r)-1\right|$ are arbitrarily small. Hence we may assume that $g \in N$ and $1 /(2 \ell-1) \pi<\varepsilon_{1}$.

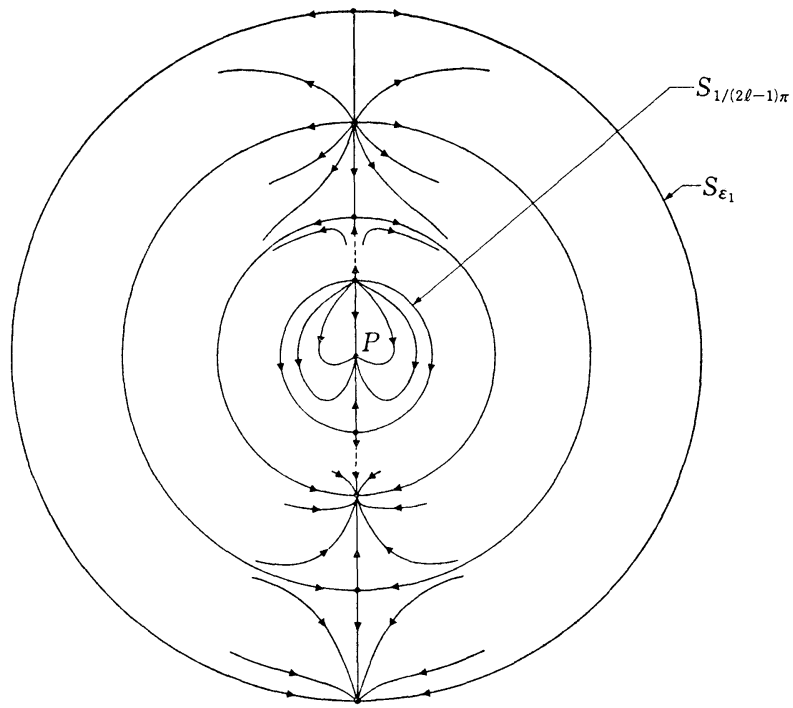

Fig. 6.

If $y$ is a fixed point of $f$ satisfying $\|y\|<\varepsilon_{1} / 2$ then $\tau^{-1}(y)$ contains a fixed point of $g$. In fact, since $\tau g=f \tau, \tau^{-1}(y)$ is a g-invariant closed subset. By the condition (a) above, each $x$ in $\tau^{-1}(y)$ satisfies

$$
\begin{aligned}
\|x\| & \leqq\|y\|+\|y-x\| \\
& =\|y\|+\|\tau x-x\| \\
& <\frac{\varepsilon_{1}}{2}+\frac{\varepsilon_{1}}{2}=\varepsilon_{1} .
\end{aligned}
$$

Any invariant closed subset of $g$, included in $B_{\varepsilon_{1}}$, contains at last one fixed point (see Fig. 6). Hence, for each fixed point $y$ of $f$ in $B_{\epsilon_{1} / 2}$, there 
is a fixed point $x$ of $g$ such that $\tau(x)=y$ and $x \in B_{\varepsilon_{1}}$. There are infinitely many fixed points of $f$ in $B_{\varepsilon_{1} / 2}$, but there are at most finite fixed points of $g$ in $B_{\varepsilon_{1}}$. This is a contradiction. Therefore $f$ is topologically unstable in $\operatorname{Diff}^{1}(M)$.

\section{REFERENCES}

[1] M. C. Irwin, Smooth dynamical systems, Academic press, 1980.

[2] A. Morimoto, The method of pseudo-orbit tracing and stability of dynamical systems, Seminar Note 39, Dept. of Math. Tokyo Univ., 1979, (in Japanese).

[3] J. Palis and S. Smale, Structural stability theorems, Proceedings of symposia in pure math. (Global Analysis), Amer. Math. Soc., 14 (1970), 223-231.

[4.] C. Robinson, Structural stability of $C^{1}$ diffeomorphisms, J. Differential Equations, 22 (1976), 28-73.

[5] S. Smale, Morse inequalities for a dynamical system, Bull. Amer. Math. Soc., 66 (1960), 43-49.

[6] F. Takens, On Zeeman's tolerance stability conjecture, Manifolds-Amsterdam 1970, Lecture Notes in Math., Springer, 197 (1971), 209-219.

[ 7 ] - Tolerance stability, Dynamical Systems-Warwick 1974. Proceedings 1973/74 (Edited by A, Manning), Lecture Notes in Math., Springer, 468 (1975), 293-304.

[ 8 ] W. White, On the tolerance stability conjecture, Dynamical systems (Edited by M. Peixoto), Academic Press, 1973, 663-665.

Department of Mathematics

Faculty of General Education

Nagoya University

Chikusa-ku, Nagoya

464, Japan 